\section{Polymicrobial Diseases}

\section{K.A. Brogden}

and J.M. Guthmiller, editors

\author{
ASM Press \\ Washington, D.C., 2002 \\ ISBN: 1-55581-244-9
}

Pages: 446, Price: $\$ 115.95$

Polymicrobial diseases involve multiple infectious agents and are referred to as complex, complicated, mixed, dual, secondary, synergistic, concurrent, polymicrobial, coinfections. This new book, a collection of 21 chapters written by a variety of authors, reviews mixed infections in animals and humans. The chapters are gathered into sections on polyviral diseases, polybacterial diseases, viral and bacterial infections, fungal infections, infections resulting from microbe-induced immunosuppression, and a concluding perspective. Polymicrobial diseases described include abscesses, AIDS-related opportunistic infections, conjunctivitis, gastroenteritis, hepatitis, multiple sclerosis, otitis media, periodontal diseases, respiratory diseases, and genital infections. Approximately two-thirds of the chapters deal with human diseases; the others discuss infections in cattle, goats, and pigs.

The chapters are generally well written with a focus on microbiology, pathogenesis, and to a lesser degree, treatment. The chapters on abscesses, multiple sclerosis, and mixed mycotic infections are especially informative. The chapter on abscesses provides a comprehensive review of the microbiology processes involved, the role of anaerobes in mixed infections, and animal models. The section on viruses and multiple sclerosis is provocative in its proposal that several viruses might coexist and interact to promote multiple sclerosis and other neurologic diseases. The list of candidate etiologic agents includes Human herpesvirus-6, human T-lymphotropic virus type 1, measles viruses, JC virus, Epstein-Barr virus, and herpes simplex virus-1. The chapter on mixed mycotic infections adequately discusses how fungi interact by mechanisms such as commensalism, opportunism, mixed colonization, coisolation, and dual and polymicrobial infection.

Growing two or more microbes in the laboratory in a clinical situation does not prove that a polymicrobial infection is the cause of the disease. The editors and authors do not provide a framework similar to that of Robert Koch or Bradford Hill, which one can use to decipher the role(s) of each candidate agent in a polymicrobial disease. A limited discussion is provided on the role of noninfectious factors, such as genetics of the host, retained "hardware," alcohol in hepatitis, or tobacco use in respiratory diseases. How each of the chapters was selected for inclusion and what other topics were considered is not clear.

The reference lists are one of the book's strengths but also a weakness. The lists are extensive, occupying about $30 \%$ of the book's pages. Prioritizing the outside readings on each topic would have been useful. Several of the chapters might have been combined, such as the two on periodontal diseases, those on retroviruses, and those on respiratory diseases in humans, cattle, and pigs. In the next edition, the authors might explore the polymicrobial etiology of Reye syndrome, autoimmune disorders, athero- sclerosis, and cancers, such as Kaposi sarcoma, hepatocellular sarcoma, and cervical cancer. I recommend the book to those who think beyond the "single agent, single disease" framework and imagine multifactorial causes for those diseases currently listed as "etiology unknown."

\section{Harry W. Haverkos}

Food And Drug Administration, Rockville, Maryland

\section{Manual of Commercial Methods in Clinical Microbiology}

\author{
Allan L. Truant \\ American Society \\ for Microbiology \\ Washington, D.C., 2002 \\ ISBN: 1-55581-189-2
}

Pages: 502, Price: $\$ 115.95$

During the past 25-30 years, tremendous strides have been made in the development of various commercial methods designed to simplify the isolation (in some cases) and the detection or identification (in most cases) of many different microbes in the laboratory. During these years, the time-honored conventional test methods have served the overall science of

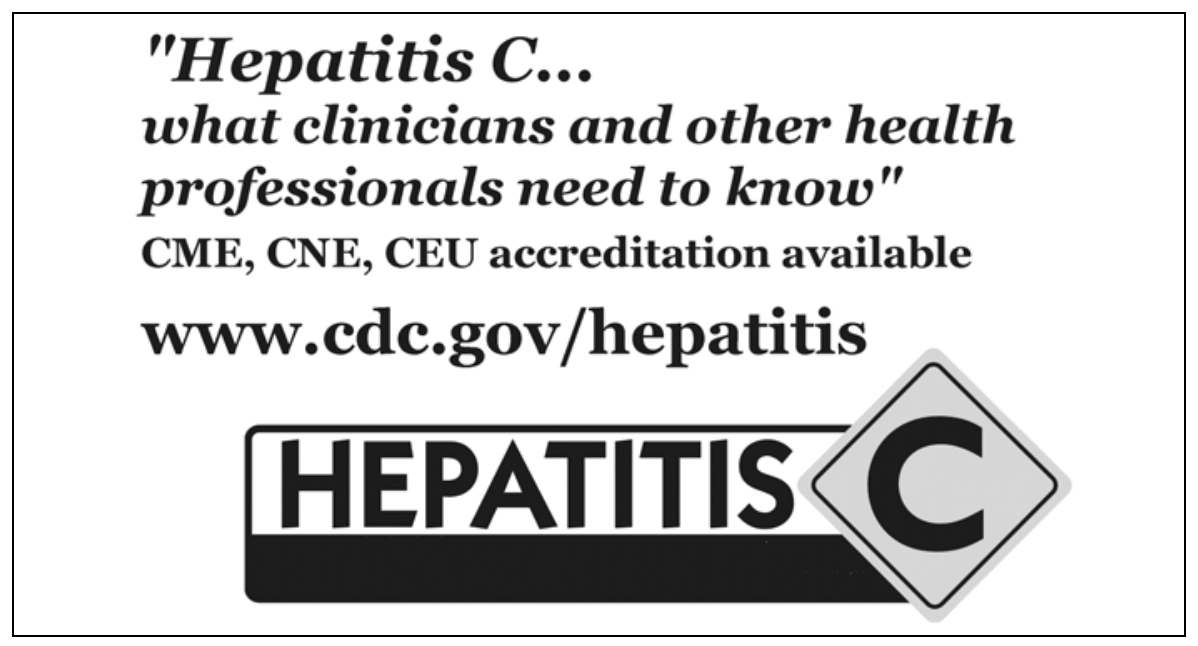


microbiology well. However, in a clinical microbiology laboratory, speed and accuracy are essential because the specimen must be processed and the results returned to the requesting physician by yesterday, if possible. Thus, many of the commercial methods reviewed in this book were developed mainly for use in the clinical microbiology laboratory, providing both rapid and accurate results with a minimum of hands-on use.

To my knowledge, this reference manual is the first resource that covers all subdisciplines of clinical microbiology. The book contains 18 chapters, including separate chapters on molecular microbiology, emerging infectious diseases, information management, and veterinary clinical microbiology, as well as chapters on bacterial identification and antimicrobial susceptibility testing, blood cultures, mycology and mycobacteriology, virology, and parasitology. A chapter on licensure and regulation of commercial products is also included, which I found helpful. In addition, the book provides an appendix that lists the manufacturers and distributors for many of the systems described in the book. The authors include a description of the sensitivities, specificities, and predictive values of the tests from peer-reviewed sources. Another chapter of interest focuses on future technology for the clinical microbiology laboratory. My only suggestion is that future editions of this text include a chapter on the history of how all these commercial tests came into being, instead of various authors alluding to this point in their respective chapters.

Each chapter is well referenced, and many chapters contain tabular material that is, for the most part, easy to read and understand. The photography is adequate, although several photographs are blurred and lack clear definition. Although the intended audience for this book is primarily clinical microbiologists and other professionals who work in these environments, I suspect that many physicians, including infectious disease specialists, will find this book especially valuable when deciding what tests to order for their patients, especially in light of the high costs of health care.

As with any multi-authored text, some unevenness in the writing is expected. However, I believe that the overall scope and format of this book are quite useful, and that readers will find this manual a valuable, comprehensive source of information. The authors are to be commended for tackling such an enormous project and successfully presenting it in such a readable format.

\section{William J. Martin}

Tucson, Arizona
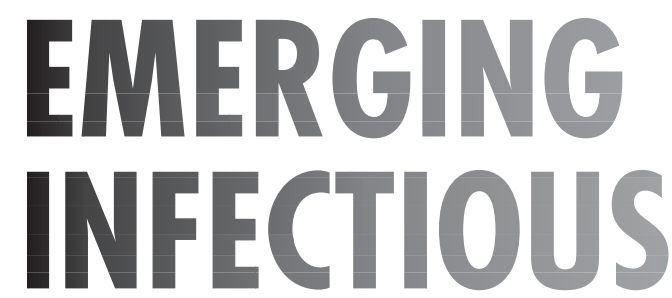

\section{Full text free online at} www.cdc.gov/eid

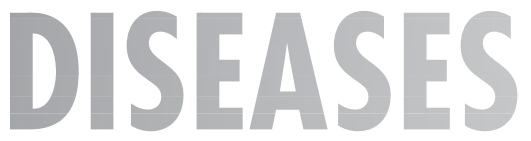

The print journal is available at no charge to public health professionals

YES, I would like to receive Emerging Infectious Diseases.

Please print your name and business address in the box and return by fax to $404-371-5449$ or mail to EID Editor CDC/NCID/MS D61 1600 Clifton Road, NE Atlanta, GA 30333

Moving? Please give us your new address (in the box) and print the number of your old mailing label here

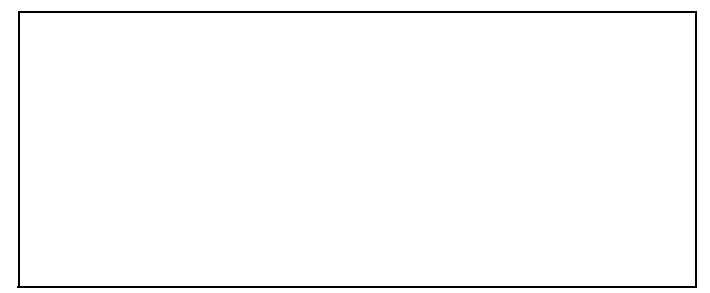

